\title{
Inhibition of the Phosphodiesterase 4 (PDE4) Enzyme Reverses Memory Deficits Produced by Infusion of the MEK Inhibitor U0I26 into the CAI Subregion of the Rat Hippocampus
}

\author{
Han-Ting Zhang*,', Yu Zhao', Ying Huang', Nandakumar R Dorairaj', L Judson Chandler ${ }^{3}$ \\ and James $M$ O'Donnell' \\ 'Department of Pharmacology, The University of Tennessee Health Science Center, Memphis, TN, USA; ${ }^{2}$ Department of Pharmacology, \\ Louisiana State University Health Sciences Center, Shreveport, LA, USA; ${ }^{3}$ Departments of Physiology/Neuroscience and Psychiatry, Medical \\ University of South Carolina, Charleston, SC, USA
}

\begin{abstract}
Cyclic AMP-specific phosphodiesterase 4 (PDE4), which is an integral component of NMDA receptor-mediated cAMP signaling, is involved in the mediation of memory processes. Given that NMDA receptors also mediate MEK/mitogen-activated protein kinase (MAPK, ERK) signaling, which is involved in synaptic plasticity, and that some PDE4 subtypes are phosphorylated and regulated by ERK, it was of interest to determine if PDE4 is involved in MEK/ERK signaling-mediated memory. It was found that rolipram, a PDE4-selective inhibitor, reversed the amnesic effect in the radial-arm maze test of the MEK inhibitor U0 I 26 administered into the CAI subregion of the rat hippocampus. Consistent with this, rolipram, either by peripheral administration or direct intra-CAI infusion, enhanced the retrieval of long-term memory impaired by intra-CAI infusion of $\cup 0126$ using the step-through inhibitory avoidance test. The same dose of rolipram did not affect U0I26-induced reduction of phospho-ERKI/2 levels in the CAI subregion. However, in primary cultures of rat cerebral cortical neurons, pretreatment with $\cup 0126$ increased PDE4 activity; this was correlated with the U0I26-induced reduction of phosphoERKI/2 levels. These results suggest that MEK/ERK signaling plays an inhibitory role in regulating PDE4 activity in the brain; this may be a novel mechanism by which MEK/ERK signaling mediates memory. PDE4 is likely to be an important link between the CAMP/PKA and MEK/ERK signaling pathways in the mediation of memory.

Neuropsychopharmacology (2004) 29, 1432-1439, advance online publication, 28 April 2004; doi: 10.1038/sj.npp. 1300440
\end{abstract}

Keywords: phosphodiesterase 4 (PDE4); mitogen-activated protein kinase (ERK); memory; hippocampus; rolipram; U0I26

\section{INTRODUCTION}

Cyclic AMP (cAMP)-specific phosphodiesterase 4 (PDE4), which catalyzes hydrolysis of cAMP, plays a critical role in the control of intracellular cAMP concentrations (Houslay, 2001). PDE4 has been shown to be an important mediator of memory processes associated with $N$-methyl-D-aspartate (NMDA) receptor-mediated signaling. Pretreatment with rolipram, a PDE4-selective inhibitor, reverses the amnesic effect of the NMDA receptor antagonist MK-801 in rats and enhances NMDA-induced increases in cAMP in rat cerebral

*Correspondence: Dr H-T Zhang, Department of Pharmacology, The University of Tennessee Health Science Center, 874 Union Ave. Memphis, TN 38163, USA, Tel: 901448 3547, Fax: 90। 4483849,

E-mail: hzhang@utmem.edu

Received 6 January 2004; revised 9 February 2004; accepted II February 2004

Online publication: 16 February 2004 at http://www.acnp.org/citations/ Npp02160404004/default.pdf cortical neurons (Zhang et al, 2000; Suvarna and O'Donnell, 2002). This is supported by recent findings that rolipram blocks memory deficits induced by heterozygous deficiency of cAMP-responsive element binding protein (CREB)binding protein (CBP; Bourtchouladze et al, 2003), and that PDE4 is involved in the induction of long-term potentiation (LTP) in the CA1 subregion of the hippocampus (Ahmed and Frey, 2003), an area where PDE4 is highly expressed (McPhee et al, 2001). These results indicate that PDE4 in the hippocampus may play an important role in cAMP signaling in the mediation of memory processes.

Cyclic AMP/protein kinase A (PKA) signaling has been shown to mediate synaptic plasticity and memory (Frey et al, 1993; Izquierdo et al, 2002). Inhibition of cAMP/PKA signaling impairs memory, especially long-term memory (Koh et al, 2002); stimulation of this pathway enhances memory (Barad et al, 1998; Zhang et al, 2000). Similar to cAMP signaling, the MEK/mitogen-activated protein kinase (MAPK, ERK) pathway also has been shown to be involved 
in the mediation of synaptic plasticity and memory (Adams and Sweatt, 2002; Schafe et al, 2000; Sharma et al, 2003). Activation of ERK signaling enhances the induction of longterm-synaptic facilitation as well as long-term memory (Purcell et al, 2003). Conversely, inhibition of ERK signaling in the dorsal hippocampus blocks the formation of longterm memory, but not acquisition of short-term memory (Blum et al, 1999; Sharma et al, 2003). Further, both signaling pathways interact with each other. Increases in cAMP levels stimulate or inhibit ERK signaling, depending on cell types. In PC12 or CHO-K1 cells, cAMP increases ERK activity; in C6 or NB2A cells, cAMP inhibits ERK activity (Qiu et al, 2000). Conversely, in F442A cells, activation of ERK with epidermal growth factor (EGF) increases cAMP by inhibiting a PDE4 isoenzyme (Hoffmann et al, 1999). Consistently, crosstalk exists between cAMP and ERK signaling in the CA1 subregion of the hippocampus (Patterson et al, 2001; Stork and Schmitt, 2002), which is involved in the mediation of synaptic plasticity (Patterson et al, 2001; Roberson et al, 1999) and ERK signalingmediated long-term memory (Blum et al, 1999). Further, PDE4 isoemzymes are phosphorylated and regulated by ERK2. In COS1 cells, the long-form PDE4 isoenzymes such as PDE4D3 and PDE4D5 are inhibited by ERK2 phosphorylation, whereas the short-form PDE4 such as PDE4B2 is activated by ERK2 phosphorylation (Baillie et al, 2000). While it is not known whether ERK signaling affects PDE4 activity in neuronal cells, these results indicate that PDE4 may play an essential role in crosstalk between the two signaling pathways; PDE4 inhibitors may reverse memory deficits induced by inhibition of ERK signaling. To address these questions, we examined whether rolipram would alter memory impairments produced by bilateral infusion of the MEK inhibitor U0126 into the CA1 subregion of the hippocampus. Memory was assessed in rats using the eightarm radial maze and step-through inhibitory avoidance tests. In addition, the interaction between MEK and PDE4 inhibition was assessed by Western blotting for ERK and phospho-ERK. PDE4 activity was assayed in cerebral cortical neurons treated with U0126.

\section{MATERIALS AND METHODS}

\section{Animals}

Male Sprague-Dawley rats (250-300 g) were housed individually in plastic cages in a room that was kept at a constant temperature $\left(22-23^{\circ} \mathrm{C}\right)$ and on a 12 -h on/12-h off light cycle (lights on at $0600 \mathrm{~h}$ ). Water and food were freely available, except for the rats used for the radial-arm maze task; for these rats, food was restricted to $16 \mathrm{~g}$ per day after test sessions, so as to maintain body weights at $80-85 \%$ of free-feeding weights. All experiments were carried out according to the 'NIH Guide for the Care and Use of Laboratory Animals' (revised 1996) and were approved by the Animal Care and Use Committee at the University of Tennessee Health Science Center.

\section{Surgery}

All surgery was performed under aseptic conditions. Rats were anesthetized $(50 \mathrm{mg} / \mathrm{kg}$ ketamine and $6 \mathrm{mg} / \mathrm{kg}$ xyla- zine) and placed in a stereotaxic holder (Stoelting, Wood Dale, IL). 22-Gauge guide cannulae (22-gauge) (Plastics One, Roanoke, VA) were implanted bilaterally into the CA1 subregion of the hippocampus using the following coordinates: $\mathrm{AP},-3.3 \mathrm{~mm}$ from Bregma; $\mathrm{ML}, \pm 2.0 \mathrm{~mm}$ from the midline; DV, $-2.5 \mathrm{~mm}$ from dura (Blum et al, 1999; Paxinos and Watson, 1986). At 1 week after surgery, rats resumed daily sessions (the radial-arm maze task) or started training (the step-through task). Following completion of the testing, sites of cannula implantation were verified histologically.

\section{The Radial-Arm Maze Test}

The test was performed as described previously (Zhang et al, 2000). Rats were trained twice a day to locate food pellets in each of four randomly selected arms in the eight-arm radial maze (each arm was $60 \mathrm{~cm}$ long, $10 \mathrm{~cm}$ wide, and $12 \mathrm{~cm}$ high; the maze was elevated $70 \mathrm{~cm}$ above the floor). Four parameters were recorded: (1) working memory errors, that is, entries into baited arms that had already been visited during the same trial; (2) reference memory errors, that is, entries into unbaited arms; (3) total arm entries; (4) the test duration, that is, the time spent in the collection of all the pellets in the maze. When the working memory error was zero and the average reference memory error was less than 1 in five successive trials, the drug tests were initiated.

Rolipram (Schering AG, Berlin, Germany) was dissolved in saline containing 1\% dimethyl sulfoxide (DMSO; for i.p. injections) or DMSO (for intra-CA1 infusions); U0126 (Promega, Madison, WI) was dissolved in DMSO. U0126 $(4 \mu \mathrm{g} / \mathrm{side})$ was infused bilaterally into the CA1 subregion of the hippocampus in a volume of $0.5 \mu \mathrm{l} /$ side in a 2 -min period using a syringe pump; the 28-gauge infusion cannulae, which were $1 \mathrm{~mm}$ longer than the guide cannulae, were left in place for an additional minute to permit diffusion. After $30 \mathrm{~min}$, this treatment was repeated along with an injection of rolipram $(0.1 \mathrm{mg} / \mathrm{kg}$, i.p.) or its vehicle. Behavioral experiments were performed $30 \mathrm{~min}$ later in a lighted, quiet room that contained several extra-maze visual cues. The frequency of working and reference memory errors was determined. The frequency was calculated as the number of working or reference memory errors divided by total number of entries into the arms.

\section{The Inhibitory Avoidance Test}

The test was performed as described previously (Zhang $\mathrm{et} \mathrm{al}$, 2000). During training, the rat was placed in the illuminated compartment of a two-compartment chamber (Model E1016SC, Coulbourn Instruments, Allentown, PA), facing away from the closed guillotine door for $1 \mathrm{~min}$, after which the door was raised. After the rat entered the darkened side, the door was closed and a $0.5 \mathrm{~mA}$ electric foot-shock was administered for $3 \mathrm{~s}$. After $24 \mathrm{~h}$, U0126 $(4 \mu \mathrm{g} /$ side into the CA1 subregion, twice; total dose, $16 \mu \mathrm{g})$ and rolipram $(0.1 \mathrm{mg} / \mathrm{kg}$, i.p. $)$ were administered as described above. At 30 min after i.p. injection of rolipram, the rat was again placed on the illuminated side, with the guillotine door open. For intra-CA1 infusion, rolipram $(15 \mu \mathrm{g} / \mathrm{side}$; total dose, $30 \mu \mathrm{g}$ ) was given $10 \mathrm{~min}$ after the second CA1 infusion of U0126, which was given $30 \mathrm{~min}$ prior to the test. The latency for entering the darkened compartment was 
recorded for up to $180 \mathrm{~s}$. At $48 \mathrm{~h}$ after the initial training, retention was tested again without any additional treatments.

\section{Tissue Preparation and Western Blots}

CA1 tissue of the hippocampus from a separate group of rats was used for ERK1/2 activity analysis. At $30 \mathrm{~min}$ after the second CA1 infusion of U0126 plus vehicle or rolipram $(0.1 \mathrm{mg} / \mathrm{kg}$, i.p.) as described above, rats were decapitated, their brains removed, and the hippocampi dissected. Bilateral CA1 tissue was punched with a plastic pipette (inner diameter, $0.2 \mathrm{~mm}$ ), sonicated for $10 \mathrm{~s}$ in $300 \mu \mathrm{l}$ of $2 \%$ SDS, and centrifuged at $15000 \mathrm{~g}$ for $30 \mathrm{~min}$ at $4^{\circ} \mathrm{C}$. The resulting supernatant was removed, an aliquot was taken for protein assay, and the remainder was stored at $-80^{\circ} \mathrm{C}$ until use.

Gel electrophoresis and immunoblotting were performed as described previously (Chandler et al, 2001). Aliquots of the frozen supernatant were diluted with $2 \times$ electrophoresis sample buffer $(50 \mathrm{mM}$ Tris $\mathrm{HCl}, \mathrm{pH} 6.7,4 \% \mathrm{w} / \mathrm{v}$ glycerol, 4\% SDS, $1 \%$ 2-mercaptoethanol, and $0.02 \mathrm{mg} / \mathrm{ml}$ bromphenol blue) and then boiled for $10 \mathrm{~min} ; 20 \mu \mathrm{g}$ protein samples were separated using $7.5 \%$ SDS-polyacrylaminde gels and transferred to Immobilon-P membranes (Millipore, Bedford, MA). After transfer, the membranes were blocked in PBST buffer containing 5\% nonfat dried milk (Carnation) and $1 \%$ bovine serum albumin for $1 \mathrm{~h}$ at room temperature, washed and incubated overnight at $4{ }^{\circ} \mathrm{C}$ with anti-phosphoERK1/2 antibody ( $1: 1000$; Cell Signaling Technology, Inc., Beverly, MA). The membranes were then washed and incubated for $1 \mathrm{~h}$ with horseradish peroxidase-conjugated goat anti-rabbit immunoglobulin G (1:2000; Cell Signaling Technology, Inc., Beverly, MA). The antigen-antibodyperoxidase complex was detected by enhanced chemiluminescence and visualized by exposure to Hyperfilm ECL (Amersham Biosciences Corp., Piscataway, NJ). The membranes were then washed with PBST and stripped, blocked, and reprobed with anti-ERK antibody (1:1000; Cell Signaling Technology, Inc., Beverly, MA) as described above. Exposed films were analyzed and quantified by computer-assisted densitometry; comparisons between treatment conditions were carried out within individual gels/immunoblots.

\section{ERK and PDE4 Activity in Neuronal Cultures}

Cerebral cortical cultures were prepared from newborn rat pups and treated as described previously (Chandler et al, 2001). Cortical cultures were washed twice with $1.5 \mathrm{ml}$ of HEPES buffer (140 mM NaCl, $5.4 \mathrm{mM} \mathrm{KCl}, 1.8 \mathrm{mM} \mathrm{CaCl}_{2}$, $100 \mathrm{nM}$ glycine, $15 \mathrm{mM}$ glucose, and $25 \mathrm{mM}$ HEPES, $\mathrm{pH} 7.4$ ) and incubated at $37^{\circ} \mathrm{C}$ with $1 \mathrm{ml}$ HEPES buffer containing $1 \mu \mathrm{M}$ TTX. After a 30-min incubation, U0126 $(0.01,0.1,1$, or $10 \mu \mathrm{M})$ or vehicle (DMSO) was added. The cultures were allowed to incubate for an additional $30 \mathrm{~min}$ (for PDE4 assay) or $60 \mathrm{~min}$ (for Western blots) before the cells were scraped into $100 \mu \mathrm{l}$ of RIPA buffer ( $50 \mathrm{mM}$ Tris, $\mathrm{pH} 8.0$, $150 \mathrm{mM} \mathrm{NaCl}, 1 \%$ Nonidet P-40, $0.5 \%$ sodium deoxycholate, and $0.1 \%$ SDS). Homogenates from triplicate dishes were combined, sonicated ( $\sim 5 \mathrm{~s}$ ), and centrifuged at $15000 \mathrm{~g}$ for $30 \mathrm{~min}$ at $4{ }^{\circ} \mathrm{C}$. The resulting supernatant was stored at $-20^{\circ} \mathrm{C}$ for subsequent determination of phospho-ERK/ERK (as described above), PDE4 activity (Zhang et al, 2002), and protein concentration using the bicinchoninic acid assay (Pierce Biotechnology, Inc., Rockford, IL). For the PDE4 determination, the supernatant was assayed in the absence (total PDE activity) and presence (rolipram-insensitive PDE or non-PDE4 activity) of $10 \mu \mathrm{M}$ rolipram; PDE4 (rolipramsensitive PDE) activity was determined by subtracting nonPDE4 activity from total PDE activity (Zhang et al, 2002).

\section{Statistical Analysis}

All data were analyzed by one-way analyses of variance (ANOVA) followed by Newman-Keuls tests for post hoc comparisons of individual means. The $r$-values were calculated using linear regression.

\section{RESULTS}

\section{Rolipram Reversed Memory Deficit Induced by Intra- CA1 Infusion of U0126 in the Radial-Arm Maze Test}

To determine the involvement of PDE4 in ERK signalingmediated memory, the effects of rolipram, a selective PDE4 inhibitor, on radial-arm maze behavior was examined; this measures hippocampus-dependent memory. Rats were implanted with cannula guides into the CA1 subregion of the hippocampus. Histological verification showed high localization of the cannula placements within the CA1 subregion (Figure 1a, b). U0126, which was directly infused into the CA1 subregion of the hippocampus at a total dose of $16 \mu \mathrm{g}(4 \mu \mathrm{g} /$ side bilaterally, two infusions), produced a significant increase in reference memory errors $(p<0.01)$. This effect of U0126 was antagonized by rolipram in a dosedependent manner $\left(\mathrm{F}_{3,29}=5.69, p<0.01\right.$; Figure $\left.2 \mathrm{a}\right)$. The treatments did not significantly affect the working memory errors (Figure 2b) or alter the exploration time except for the higher dose of rolipram (plus U0126), which increased the exploration time $(p<0.05$; Figure $2 c)$. The latter is consistent with a previous study (Zhang et al, 2000). In addition, the same doses of rolipram alone did not affect the radial-arm maze performance (data not shown). These results indicate that rolipram specifically enhances longterm memory impaired by U0126.

\section{Rolipram Enhanced Retrieval of Long-Term Memory Impaired by U0126 Infused into Hippocampal CA1 in the Step-Through Inhibitory Avoidance Test}

To confirm the effects of rolipram on the U0126-induced memory deficit, the step-through inhibitory avoidance test was used to assess retrieval of long-term memory; this also measures hippocampus-dependent memory. Rolipram was given either i.p. or bilaterally into the CA1 region of the hippocampus. At $24 \mathrm{~h}$ after initial training, U0126-treated rats did not show a significant reduction of retention because of the relatively low baseline of the vehicle control. However, rolipram treatment, either i.p. or intra-CA1, markedly increased the retention (Figure $3 \mathrm{a}, \mathrm{b}$ ). At $48 \mathrm{~h}$ after training, vehicle-treated rats almost completely recovered from memory impairment; U0126-treated rats, however, showed a significant reduction of retention. Rolipram, at a dose of $0.1 \mathrm{mg} / \mathrm{kg}$, i.p., reversed the amnesic 
a

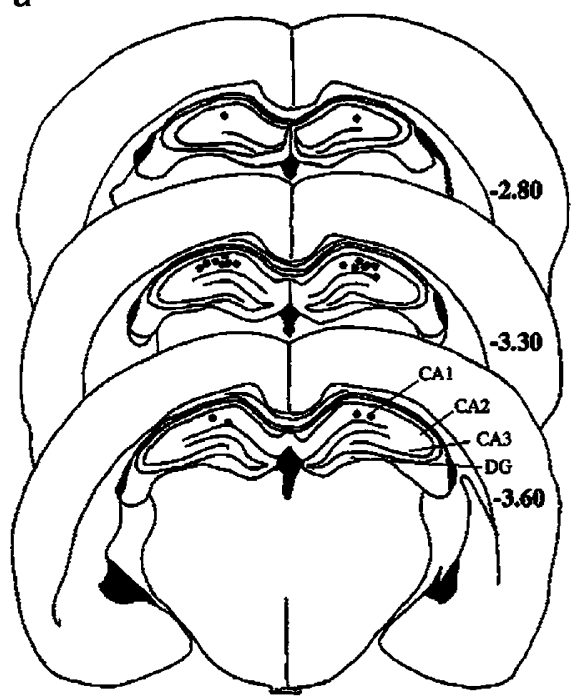

b

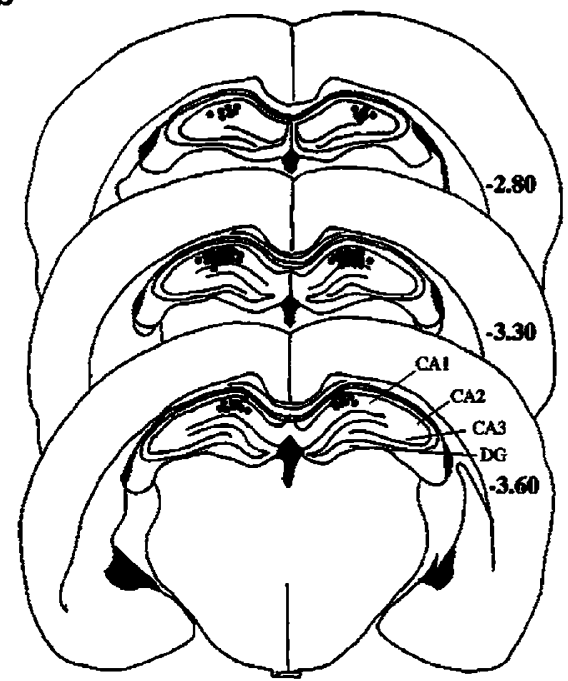

Figure I Infusion sites for the bilateral hippocampal CAI drug administration in (a) the radial-arm maze and (b) the step-through avoidance tests. Coronal sections of the rat brain at $-2.8,-3.3$, and $-3.6 \mathrm{~mm}$ from Bregma (Paxinos and Watson, 1986) are shown with CAI -3 subregions and dentate gyrus (DG) labeled.

effect of U0126 $\left(\mathrm{F}_{2,24}=4.51, p<0.05\right.$; Figure 3a). A similar antagonism was observed when rolipram $(15 \mu \mathrm{g} /$ side $)$ was administered directly into the CA1 subregion of the hippocampus $\left(\mathrm{F}_{2,12}=7.12, p<0.01\right.$; Figure $\left.3 \mathrm{~b}\right)$.

\section{Rolipram Did Not Alter U0126-Induced Reduction of Phospho-ERK Levels in the CA1 Subregion}

The behavioral data indicate a functional link between ERK and cAMP signaling pathways in the mediation of long-term memory, in which PDE4 is likely to be involved. In order to investigate this possible interaction, the phospho-ERK level was examined in the CA1 subregion of the hippocampus of rats treated with U0126 alone or in combination with doses of rolipram that were effective in the behavioral tests. Bilateral infusion of U0126 into the CA1 subregion caused a
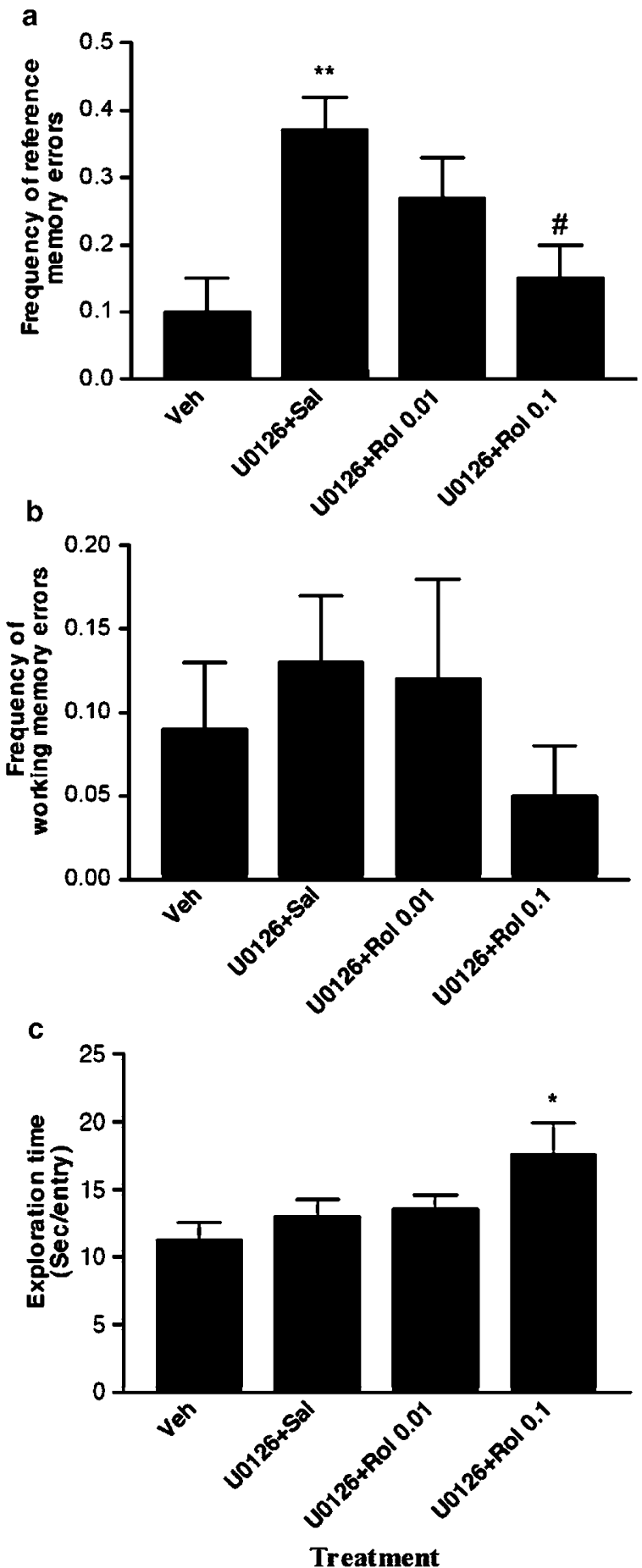

Figure 2 UOI26-induced memory deficit was antagonized by rolipram (Rol) in the radial-arm maze test in rats. (a) U0I26 significantly increased the frequency of reference memory errors; this effect was antagonized by rolipram. (b) U0I26 alone or with rolipram did not significantly alter the frequency of working memory errors. (c) The treatments did not alter the average exploration time, except U0 26 plus $0.1 \mathrm{mg} / \mathrm{kg}$ rolipram, which increased the exploration time. U0I26 (4 $\mu \mathrm{g} / \mathrm{side}$, bilateral CAI infusion twice; total, $16 \mu \mathrm{g})$ and rolipram $(0.01$ and $0.1 \mathrm{mg} / \mathrm{kg}$, i.p.) were given as described in Materials and Methods. Data shown are means \pm SE from 10 rats. ANOVA and post hoc Newman-Keuls tests revealed the following differences: $*<<0.05$, ** $p<0.01$ vs the vehicle (Veh; DMSO + saline)treated group; \# $p<0.05$ vs the $\mathrm{U} 0126$ + saline group. 
significant decrease in the levels of both phospho-ERK1 (P44 phospho-ERK; $F_{2,15}=37.22, p<0.0001$ ) and phosphoERK2 (P42 phospho-ERK; $F_{2,15}=21.31, p<0.0001$; Figure $4 \mathrm{a}, \mathrm{c}, \mathrm{d})$; by contrast, total ERK levels were not changed
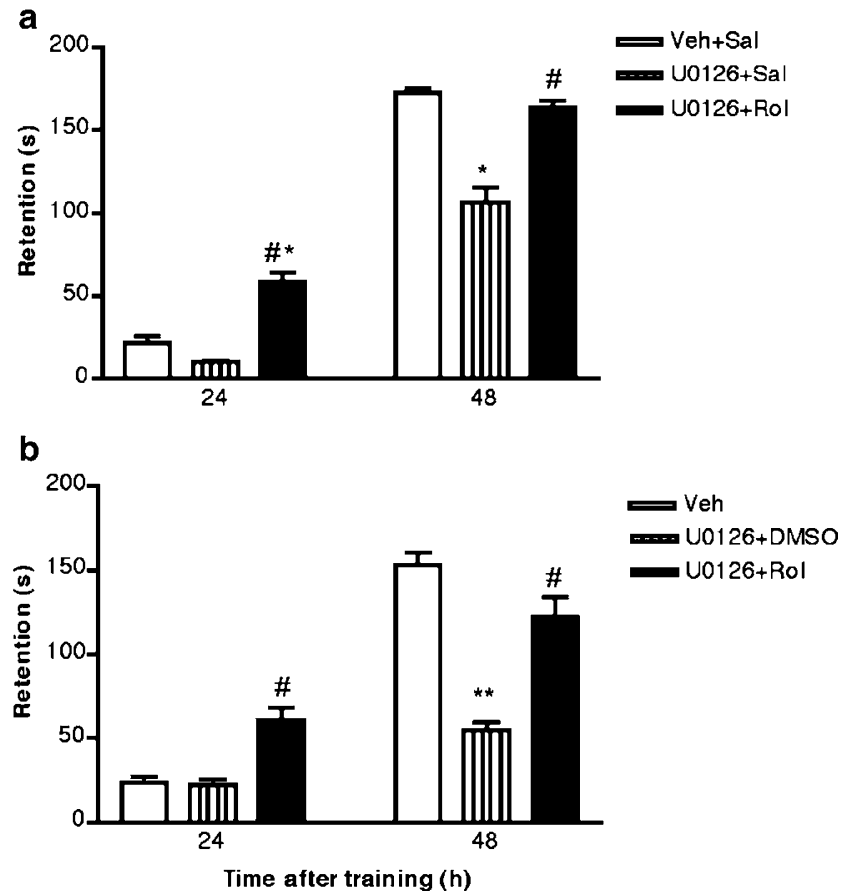

Figure 3 U0I26-induced memory deficit was antagonized by rolipram (Rol) in the step-through inhibitory avoidance test. (a) Peripheral administration of rolipram (i.p.) reversed $\mathrm{U} 0 \mathrm{I} 26$-induced reduction of retention. (b) Intra-CAI infusion of rolipram reversed U0I26-induced reduction of retention. Rats were treated with $\mathrm{UO} I 26$ and rolipram $24 \mathrm{~h}$ after initial training; test data shown were obtained 24 and $48 \mathrm{~h}$ posttraining. Administration of both drugs was the same as described above, except that of rolipram (I5 $\mu \mathrm{g} / \mathrm{side}$, bilateral CAI infusions), which was given 10 min after the second CAI infusion of U0I26. Data shown are means \pm SE from five to nine rats for each group. ANOVA and post hoc Newman-Keuls tests revealed the following differences: * $p<0.05$, *** $p<0.0$ I vs the corresponding vehicle-treated group; $\# p<0.05$ vs the corresponding $\mathrm{U} 0 \mathrm{I} 26+$ saline/DMSO group.
(Figure 4b, e). Rolipram did not alter U0126-induced inhibition of phospho-ERK1/2 in the CA1 subregion, indicating that the effect of rolipram on U0126-impaired memory did not result from a direct effect on ERK activation.

\section{U0126 Increased PDE4 Activity}

Previous work has shown that ERK activation can result in a phosphorylation-mediated inhibition of PDE4 activity in F442A cells (Hoffmann et al, 1999); however, it was not known if such a mechanism was active in neurons. In order to determine whether MEK inhibition alters PDE4 activity, the effects of U0126 on phospho-ERK levels and PDE4 activity in primary cultures of rat cerebral cortical neurons were examined. U0126 $(0.01-10 \mu \mathrm{M})$ reduced the levels of both phospho-ERK1 and phospho-ERK2 in the neurons in a concentration-dependent manner $\left(\mathrm{F}_{4,15}=31.69, p<0.0001\right.$ and $\mathrm{F}_{4,15}=14.4, p<0.0001$, respectively), without altering the total protein levels of either ERK1 or ERK2 $\left(\mathrm{F}_{4,15}=0.1\right.$, $p=0.98$ and $\mathrm{F}_{4,15}=0.08, p=0.99$, respectively; Figure $5 \mathrm{a}, \mathrm{b}$ ). The same concentrations of U0126 that reduced phosphoERK increased PDE4 activity in the neurons in a concentration-dependent manner $\left(\mathrm{F}_{4,26}=8.55, p<0.0005\right.$; Figure 5c). The U0126-induced increase in PDE4 activity was negatively correlated with the U0126-induced reduction of phospho-ERK1 and phospho-ERK2 levels $(r=0.92$ and $r=0.96$, respectively; Figure $5 \mathrm{~d}$ ). These results support the idea that ERK plays an inhibitory role in PDE4 activity in neurons.

\section{DISCUSSION}

Recent studies have shown that inhibition of hippocampal MEK/ERK signaling impairs synaptic plasticity as well as long-term memory formation (Davis et al, 2000; Kelly et al, 2003; Adams and Sweatt, 2002). Consistent with this, in the present study, direct infusion of U0126, a specific MEK inhibitor (Hotokezaka et al, 2002), into the CA1 subregion of the hippocampus, also impaired reference memory in the a

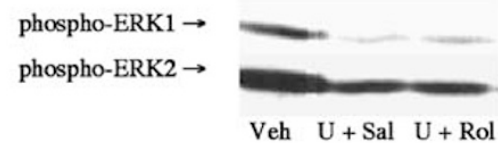

b

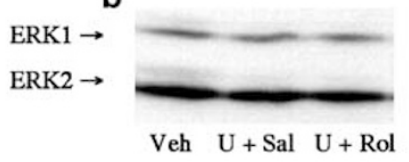

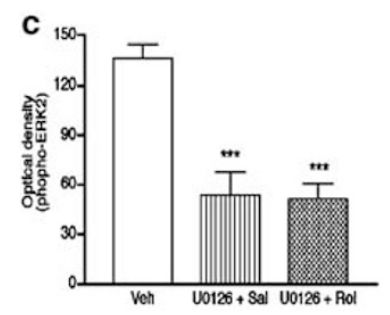
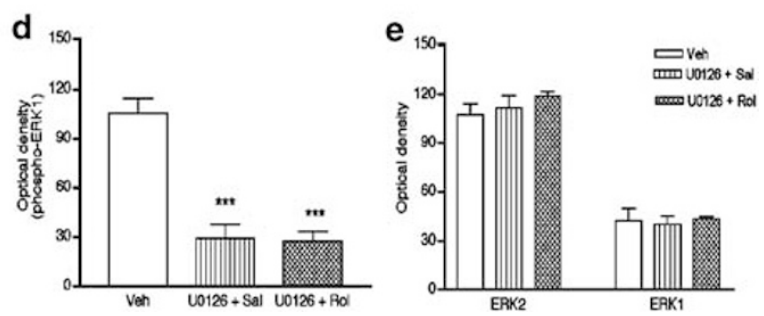

Figure 4 Rolipram did not alter U0126-induced reduction of phospho-ERK in the CAI subregion of the rat hippocampus. (a) Representative immunoblots of phospho-ERKI/2 immunoreactivity. (b) Representative immunoblots of ERKI/2 immunoreactivity. (c-e) Quantification of phospho-ERK2, phospho-ERKI, and ERKI/2, respectively, by computer-assisted densitometry. Data shown are means \pm SE from five to seven rats for each group expressed as optical density. ANOVA and post hoc Newman-Keuls tests revealed the following difference: **** $p<0.00$ I vs the vehicle (Veh) group. 
a
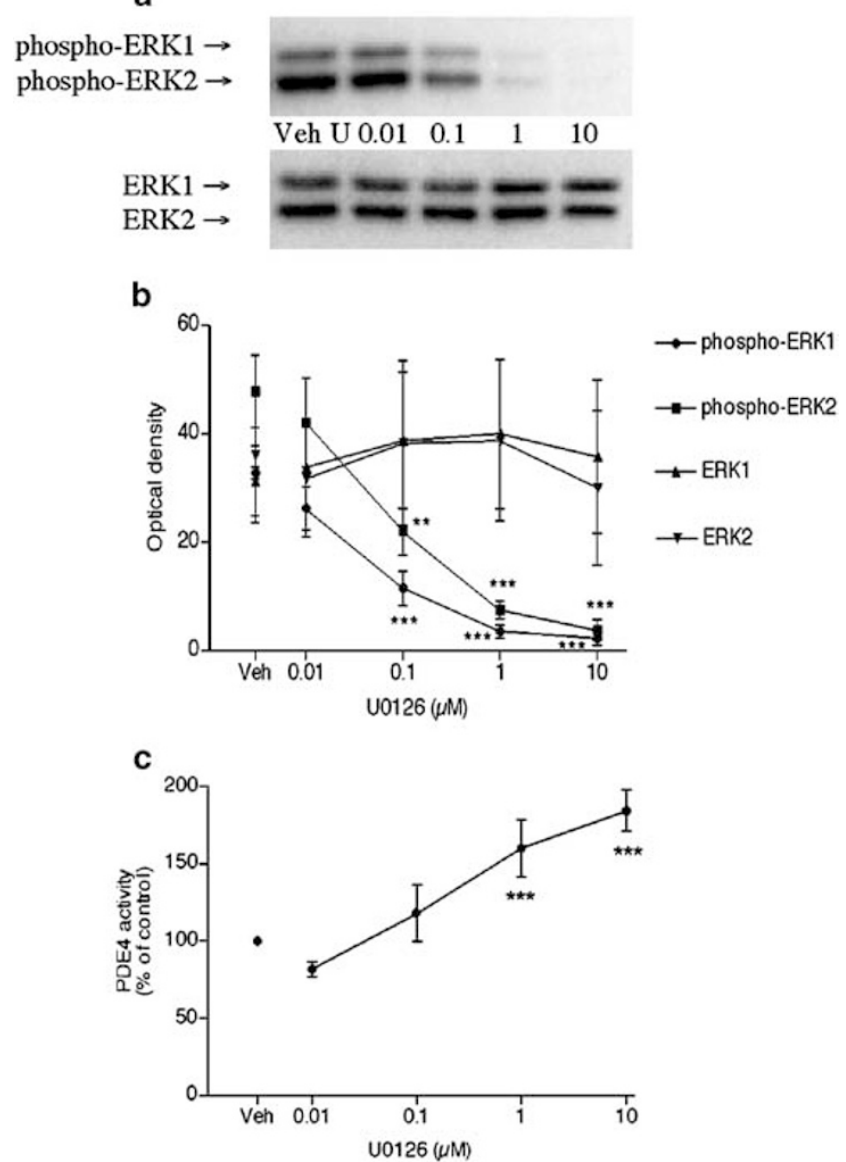

d

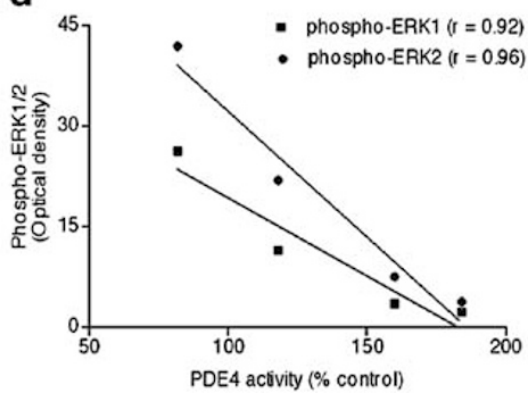

Figure 5 U0I26 decreased phospho-ERKI/2 levels and increased PDE4 activity in primary cultures of rat cerebral cortical neurons. (a) Representative immunoblots of phospho-ERKI/2 (upper panel) and ERKI/2 (lower panel) immunoreactivity. (b) Quantification of phosphoERKI/2 and ERKI/2 by computer-assisted densitometry, expressed as optical density. (c) PDE4 activity after incubation with UOI26. (d) Correlation between PDE4 activity and phospho-ERKI/2. The cultured neurons were incubated with vehicle (Veh; DMSO) or U0I26 for $30 \mathrm{~min}$ in the presence of TTX $(\mid \mu M)$, which was added 30 min prior to $\cup 0 \mid 26$. The control (Veh) PDE4 activity was $14.4 \pm 1.7 \mathrm{pmol} / \mathrm{min} / \mathrm{mg}$ protein. Data shown are means \pm SE from four experiments for each group (b) or six to -10 experiments for each group except for the 0.01 and $0.1 \mu \mathrm{M} \cup 0126$ groups, in which three experiments were used (c). ANOVA and post hoc Newman-Keuls comparisons revealed the following differences: $* * * 0.0$ I, **** $p<0.00$ I vs corresponding vehicle control.

radial-arm maze, an index of long-term memory (Zhang et al, 2000). This indicates an important role of the MEK/ ERK signaling pathway in the CA1 subregion in the longterm memory process. To confirm this, we examined the retrieval of one-trial task in step-through inhibitory avoidance. ERK signaling is required for retrieval of longterm memory. The MEK inhibitor PD098059 infused into bilateral CA1 prior to the retention test blocks retrieval of long-term memory, but not short-term memory (Barros et al, 2000; Izquierdo et al, 2000). In agreement with this, U0126 administered by CA1 infusion prior to the retention test also impaired retrieval of long-term memory even if it was given $24 \mathrm{~h}$ before the second retention test (ie 48 after training). Rolipram blocked the effect of U0126 either by peripheral administration or CA1 infusion, indicating an enhancement of retrieval of long-term memory. It is not known how U0126 impaired the retrieval of one-trial inhibitory avoidance for such a long time after administration. However, a similar effect has been reported with the MEK inhibitor PD098059, which impairs long-term memory $21 \mathrm{~h}$ after administration (Walz et al, 1999). The memoryenhancing effect of rolipram is only maintained for $60 \mathrm{~min}$ after single injection (Zhang and O'Donnell, 2000). However, rolipram, $24 \mathrm{~h}$ after injection, still blocked U0126induced impairment of memory retrieval. This could be caused by rolipram reversal of U0126-induced disinhibition of PDE4 activity (see below).

Although it is established that cAMP/PKA signaling plays an essential role in the mediation of memory (Barad et al, 1998; Frey et al, 1993; Izquierdo et al, 2002; Koh et al, 2002; Zhang et al, 2000), the involvement of MEK/ERK signaling makes the memory mediation more complicated. To date, there is no evidence for the interaction of cAMP and ERK signaling in mediating memory. Thus, it is interesting that U0126-induced memory deficits were reversed by either peripheral or intra-CA1 administration of rolipram, a specific PDE4 inhibitor. These results suggest that PDE4 in the CA1 subregion of the hippocampus plays an important role in the memory process mediated by MEK/ ERK signaling.

It was possible that rolipram reversed U0126-induced memory deficit by antagonizing ERK inhibition by U0126 and/or inhibiting PDE4 activity. However, rolipram failed to affect U0126-induced decrease in phospho-ERK levels, indicating that it did not enhance memory via alteration of ERK activity. It is reported that rolipram enhances memory via inhibition of PDE4 and a subsequent increase in the cAMP concentration (Barad et al, 1998; Zhang et al, 2000). This appears to be the only known mechanism underlying the memory enhancement by rolipram. Therefore, PDE4 appears to be a connection between cAMP and ERK signaling pathways in mediating memory. This is further supported by in vitro studies. In COS-1 cells, ERK2 activation induces a phosphorylation-mediated inhibition of activity of transfected PDE4D3 and PDE4B1 (Baillie et al, 2000; Hoffmann et al, 1999). This mechanism also appears to be active in neurons since it was demonstrated that MEK inhibition increased PDE4 activity in cerebral cortical neurons. The change in PDE4 activity was correlated with inhibition of ERK1/2, quantified by phospho-ERK1/2 levels. This suggests an inhibitory role of ERK signaling in PDE4 activity.

Mammalian PDE4 enzymes are encoded by four genes: PDE4A, PDE4B, PDE4C, and PDE4D (Houslay, 2001). While PDE4C is minimally expressed in the central nervous system, the other three PDE4 subtypes are highly expressed 
thoughout the brain (Cherry and Davis, 1999; Perez-Torres et al, 2000). However, the CA1 subregion of the rat hippocampus appears to express PDE4A and PDE4D at the highest levels, while PDE4B is expressed only at a low level in this structure (Perez-Torres et al, 2000). Technical limitations prevented determining PDE4 activity in CA1 neurons, so primary cultures of cerebral cortical neurons, which express PDE4A, PDE4B, and PDE4D, were used (D'Sa et al, 2002). Since activation of ERK phosphorylates PDE4B, PDE4C, and PDE4D, but not PDE4A (Baillie et al, 2000), it appears that PDE4A is not a component of U0126stimulated PDE4 activity. In addition, ERK phosphorylation results in inhibition of only long-form PDE4 variants, but causes activation of short-form variants (MacKenzie et al, 2000; O'Donnell and Zhang, 2004). Finally, PDE4 activity in the hippocampus is primarily attributed to PDE4D (Jin et al, 1999; Zhang et al, 2002), suggesting that changes in PDE4 activity induced by ERK-mediated phosphorylation involve this subtype to the greatest extent. Given that the shortform PDE4D variants are either somewhat activated (PDE4D1) or not affected (PDE4D2 and PDE4D6) by ERK activation (MacKenzie et al, 2000), the long-form PDE4Ds, including PDE4D3, 4D4, 4D5, and 4D7 (Wang et al, 2003; O'Donnell and Zhang, 2004), could be the targets of ERK phosphorylation.

Taken together, the present results suggest that PDE4, as a critical regulator in the control of intracellular cAMP concentrations, provides a connection between cAMP/PKA and MEK/ERK signaling pathways. Given that inhibition of MEK/ERK signaling by U0126 increased PDE4 activity in neurons, MEK/ERK signaling may exert an inhibitory action on PDE4, which plays a major role in ERK-mediated memory processes. PDE4 inhibitors can reverse memory deficits induced by inhibition of either cAMP/PKA signaling or MEK/ERK signaling.

\section{ACKNOWLEDGEMENTS}

This work was supported by research grants (MH40694, MH51175) and an Independent Scientist Award (MH01231) from the National Institute of Mental Health and a research grant from the National Institute on Alcohol Abuse and Alcoholism (AA10983). We thank Mr Robert C Moore and Ms Kathleen Mishler for their assistance.

\section{REFERENCES}

Adams JP, Sweatt JD (2002). Molecular psychology: roles for the ERK MAP kinase cascade in memory. Annu Rev Pharmacol Toxicol 42: 135-163.

Ahmed T, Frey JU (2003). Expression of the specific type IV phosphodiesterase gene PDE4B3 during different phases of longterm potentiation in single hippocampal slices of rats in vitro. Neuroscience 117: 627-638.

Baillie GS, MacKenzie SJ, McPhee I, Houslay MD (2000). Subfamily selective actions in the ability of Erk2 MAP kinase to phosphorylate and regulate the activity of PDE4 cyclic AMPspecific phosphodiesterases. Br J Pharmacol 131: 811-819.

Barad M, Bourtchouladze R, Winder DG, Golan H, Kandel E (1998). Rolipram, a type IV-specific phosphodiesterase inhibitor, facilitates the establishment of long-lasting long-term potentiation and improves memory. Proc Natl Acad Sci USA 95: 15020-15025.
Barros DM, Izquierdo LA, Mello e Souza T, Ardenghi PG, Pereira $\mathrm{P}$, Medina JH et al (2000). Molecular signalling pathways in the cerebral cortex are required for retrieval of one-trial avoidance learning in rats. Behav Brain Res 114: 183-192.

Blum S, Moore AN, Adams F, Dash PK (1999). A mitogen-activated protein kinase cascade in the CA1/CA2 subfield of the dorsal hippocampus is essential for long-term spatial memory. J Neurosci 19: 3535-3544.

Bourtchouladze R, Lidge R, Catapano R, Stanley J, Gossweiler S, Romashko D et al (2003). A mouse model of Rubinstein-Taybi syndrome: defective long-term memory is ameliorated by inhibitors of phosphodiesterase 4. Proc Natl Acad Sci USA 100: 10518-10522.

Chandler LJ, Sutton G, Dorairaj NR, Norwood D (2001). N-methyl $\mathrm{D}$-aspartate receptor-mediated bidirectional control of extracellular signal-regulated kinase activity in cortical neuronal cultures. J Biol Chem 276: 2627-2636.

Cherry JA, Davis RL (1999). Cyclic AMP phosphodiesterases are localized in regions of the mouse brain associated with reinforcement, movement, and affect. J Comp Neurol 407: 287-301.

Davis S, Vanhoutte P, Pages C, Caboche J, Laroche S (2000). The MAPK/ERK cascade targets both Elk-1 and cAMP response element-binding protein to control long-term potentiationdependent gene expression in the dentate gyrus in vivo. J Neurosci 20: 4563-4572.

D'Sa C, Tolbert LM, Conti M, Duman RS (2002). Regulation of cAMP-specific phosphodiesterases type 4B and 4D (PDE4) splice variants by cAMP signaling in primary cortical neurons. J Neurochem 81: 745-757.

Frey U, Huang YY, Kandel ER (1993). Effects of cAMP simulate a late stage of LTP in hippocampal CA1 neurons. Science 260: 1661-1664.

Hoffmann R, Baillie GS, MacKenzie SJ, Yarwood SJ, Houslay MD (1999). The MAP kinase ERK2 inhibits the cyclic AMP-specific phosphodiesterase HSPDE4D3 by phosphorylating it at Ser579. EMBO J 18: 893-903.

Hotokezaka H, Sakai E, Kanaoka K, Saito K, Matsuo K, Kitaura H et al (2002). U0126 and PD98059, specific inhibitors of MEK, accelerate differentiation of RAW264.7 cells into osteoclast-like cells. J Biol Chem 277: 47366-47372.

Houslay MD (2001). PDE4 cAMP-specific phosphodiesterases. Prog Nucleic Acid Res Mol Biol 69: 249-315.

Izquierdo LA, Barros DM, Ardenghi PG, Pereira P, Rodrigues C, Choi $\mathrm{H}$ et al (2000). Different hippocampal molecular requirements for short- and long-term retrieval of one-trial avoidance learning. Behav Brain Res 111: 93-98.

Izquierdo LA, Barros DM, Vianna MR, Coitinho A, deDavid e Silva $\mathrm{T}$, Choi $\mathrm{H}$ et al (2002). Molecular pharmacological dissection of short- and long-term memory. Cell Mol Neurobiol 22: 269-287.

Jin SLC, Richard FJ, Kuo WP, D'Ercole AJ, Conti M (1999). Impaired growth and fertility of cAMP-specific phosphodiesterase PDE4D-deficient mice. Proc Natl Acad Sci USA 96: 11998-12003.

Kelly A, Laroche S, Davis S (2003). Activation of mitogen-activated protein kinase/extracellular signal-regulated kinase in hippocampal circuitry is required for consolidation and reconsolidation of recognition memory. J Neurosci 23: 5354-5360.

Koh MT, Thiele TE, Bernstein IL (2002). Related articles, inhibition of protein kinase A activity interferes with long-term, but not short-term, memory of conditioned taste aversions. Behav Neurosci 116: 1070-1074.

MacKenzie SJ, Baillie GS, McPhee I, Bolger GB, Houslay MD (2000). ERK2 mitogen-activated protein kinase binding, phosphorylation, and regulation of the PDE4D cAMP-specific phosphodiesterases. The involvement of $\mathrm{COOH}$-terminal docking sites and NH2-terminal UCR regions. J Biol Chem 275: 16609-16617. 
McPhee I, Cochran S, Houslay MD (2001). The novel long PDE4A10 cyclic AMP phosphodiesterase shows a pattern of expression within brain that is distinct from the long PDE4A5 and short PDE4A1 isoforms. Cell Signal 13: 911-918.

O’Donnell JM, Zhang HT (2004). Antidepressant effects of inhibitors of cyclic AMP phosphodiesterase (PDE4). Trends Pharmacol Sci 25: 158-163.

Patterson SL, Pittenger C, Morozov A, Martin KC, Scanlin H, Drake C et al (2001). Some forms of cAMP-mediated long-lasting potentiation are associated with release of BDNF and nuclear translocation of phospho-MAP kinase. Neuron 32: 123-140.

Paxinos G, Watson C (1986). The Rat Brain in Stereotaxic Coordinates, 2nd edn. Academic Press: San Diego, CA.

Perez-Torres S, Miro X, Palacios JM, Cortes R, Puigdomenech P, Mengod G (2000). Phosphodiesterase type 4 isozymes expression in human brain examined by in situ hybridization histochemistry and $[3 \mathrm{H}]$ rolipram binding autoradiography. Comparison with monkey and rat brain. J Chem Neuroanat 20: 349-374.

Purcell AL, Sharma SK, Bagnall MW, Sutton MA, Carew TJ (2003). Activation of a tyrosine kinase-MAPK cascade enhances the induction of long-term synaptic facilitation and long-term memory in Aplysia. Neuron 37: 473-484.

Qiu W, Zhuang S, von Lintig FC, Boss GR, Pilz RB (2000). Cell type-specific regulation of B-Raf kinase by cAMP and 14-3-3 proteins. J Biol Chem 275: 31921-31929.

Roberson ED, English JD, Adams JP, Selcher JC, Kondratick C, Sweatt JD (1999). The mitogen-activated protein kinase cascade couples PKA and PKC to cAMP response element binding protein phosphorylation in area CA1 of hippocampus. J Neurosci 19: 4337-4348.

Schafe GE, Atkins CM, Swank MW, Bauer EP, Sweatt JD, LeDoux JE (2000). Activation of ERK/MAP kinase in the amygdala is required for memory consolidation of pavlovian fear conditioning. J Neurosci 20: 8177-8187.
Sharma SK, Sherff CM, Shobe J, Bagnall MW, Sutton MA, Carew TJ (2003). Differential role of mitogen-activated protein kinase in three distinct phases of memory for sensitization in Aplysia. J Neurosci 23: 3899-3907.

Stork PJ, Schmitt JM (2002). Crosstalk between cAMP and MAP kinase signaling in the regulation of cell proliferation. Trends Cell Biol 12: 258-266.

Suvarna NU, O'Donnell JM (2002). Hydrolysis of $N$-methylD-aspartate receptor-stimulated cAMP and cGMP by PDE4 and PDE2 phosphodiesterases in primary neuronal cultures of rat cerebral cortex and hippocampus. J Pharmacol Exp Ther 302: 249-256.

Walz R, Roesler R, Barros DM, de Souza MM, Rodrigues C, Sant'Anna MK et al (1999). Effects of post-training infusions of a mitogen-activated protein kinase kinase inhibitor into the hippocampus or entorhinal cortex on short- and longterm retention of inhibitory avoidance. Behav Pharmacol 10: 723-730.

Wang D, Deng C, Bugaj-Gaweda B, Kwan M, Gunwaldsen C, Leonard C et al (2003). Cloning and characterization of novel PDE4D isoforms PDE4D6 and PDE4D7. Cell Signal 15: 883-891.

Zhang HT, Crissman AM, Dorairaj NR, Chandler LJ, O'Donnell JM (2000). Inhibition of cyclic AMP phosphodiesterase (PDE4). Reverses memory deficits associated with NMDA receptor antagonism. Neuropsychopharmacology 23: 198-204.

Zhang HT, Huang Y, Jin SL, Frith SA, Suvarna N, Conti M et al (2002). Antidepressant-like profile and reduced sensitivity to rolipram in mice deficient in the PDE4D phosphodiesterase enzyme. Neuropsychopharmacology 27: 587-595.

Zhang HT, O'Donnell JM (2000). Effects of rolipram on scopolamine-induced impairment of working and reference memory in the radial-arm maze tests in rats. Psychopharmaco$\log y$ (Berl) 150: 311-316. 